\title{
Síndrome compartimental bilateral de pierna tras posición de litotomía prolongada. Presentación de un caso clínico
}

\section{Bilateral Leg Compartment Syndrome After Prolonged Lithotomy Position. Clinical Case Report}

\author{
Luis Díez-Albero $^{1}$ Lorena Moril-Peñalver ${ }^{1} \quad$ Andrés Lapeña-Martínez $^{1}{ }^{\circledR}$ Fernando López-Prats ${ }^{1}$ \\ ${ }^{1}$ Servicio de Cirugía Ortopédica y Traumatología, Hospital General \\ Universitario de Elche, Alicante, España \\ Rev Chil Ortop Traumatol 2021;62(2):e153-e156. \\ Address for correspondence Luis Díez-Albero, Servicio de Cirugía \\ Ortopédica y Traumatología, Hospital General Universitario de Elche, \\ Carrer Almazara 11, Alicante 03203, España \\ (e-mail: diezalberoluis@gmail.com).
}

\section{Resumen \\ Palabras Clave \\ - síndrome compartimental \\ - litotomía \\ - perneras \\ - fasciotomía \\ - presión intracompartimental}

Introducción El síndrome compartimental es una complicación infrecuente pero severa que puede aparecer ante una posición de litotomía prolongada y el uso de perneras.

Objetivo Presentar un caso de síndrome compartimental bilateral tras posición de litotomía prolongada asociada con el uso de perneras.

Material y Métodos Paciente varón de 43 años sometido a una cirugía urológica en posición habitual de litotomía durante un periodo de 6 horas. A las 2 horas de finalizar la cirugía, el paciente presentó dolor intenso y endurecimiento de compartimento anterior y lateral de ambas piernas, junto con una elevación de la creatina quinasa sérica (valor incial de $109 \mathrm{U} / \mathrm{L}$ que se elevó hasta $7.689 \mathrm{U} / \mathrm{L}$ a las 12 horas) y una imposibilidad para la flexión dorsal pasiva de ambos tobillos. El paciente no sentía dolor en el resto de los compartimentos.

Resultados Ante la sospecha de un síndrome compartimental anterolateral, se realizó fasciotomía bilateral urgente, y se observó una disminución de la perfusión muscular y mejoría de la misma tras apertura de la fascia. Se realizaron curas periódicas cada 48 horas, y se observó viabilidad del tejido hasta su cierre definitivo a los 4 días. A las 2 semanas, el paciente presentó fatiga ligera para la deambulación sin ayuda, con tumefacción en el compartimento lateral de ambas piernas. A los 10 meses de evolución, el paciente caminaba sin ayuda y con función muscular completa.

Conclusiones El conocimiento de la asociación del síndrome compartimental y la cirugía laparoscópica prolongada es esencial para un diagnóstico precoz y un tratamiento quirúrgico inmediato, para evitar graves secuelas. Los buenos resultados de nuestro paciente se deben a la rápida actuación, ya que normalmente se suele demorar. Para evitar su aparición o disminuir su incidencia, la posición de recibido

15 de febrero de 2021

aceptado

31 de marcha de 2021
DOI https://doi.org/

$10.1055 / \mathrm{s}-0041-1735298$ ISSN 0716-4548. (c) 2021. Sociedad Chilena de Ortopedia y Traumatologia. All rights reserved.

This is an open access article published by Thieme under the terms of the Creative Commons Attribution-NonDerivative-NonCommercial-License, permitting copying and reproduction so long as the original work is given appropriate credit. Contents may not be used for commercial purposes, or adapted, remixed, transformed or built upon. (https://creativecommons.org/ licenses/by-nc-nd/4.0/)

Thieme Revinter Publicações Ltda., Rua do Matoso 170, Rio de Janeiro, RJ, CEP 20270-135, Brazil 


\begin{abstract}
Keywords

- compartment syndrome

- lithotomy

- leg loops

- fasciotomy

- intracompartmental pressure

Background Compartment syndrome is a rare but severe complication resulting from a prolonged lithotomy position and the use of leg loops.

Purpose To present a case of bilateral compartment syndrome after prolonged lithotomy position associated with the use of leg loops.

Methods A 43-year-old man underwent urological surgery in the usual lithotomy position for a 6-hour period. Two hours after the end of the surgery, the patient presented severe pain and stiffening of the anterior and lateral compartments of both legs, elevated serum creatine kinase levels (the baseline value of $109 \mathrm{U} / \mathrm{L}$ increased to $7,689 \mathrm{U} / \mathrm{L}$ at 12 hours), and inability for passive dorsiflexion of both ankles. The patient reported no pain in the other compartments.

Results Suspicion of an anterolateral compartment syndrome resulted in an urgent bilateral fasciotomy; muscle perfusion was decreased, and it improved after fascial opening. Dressings were changed every 48 hours, and tissue viability was observed until the final closure at 4 days. At two weeks, the patient presented slight fatigue when walking with no assistance, in addition to swelling in the lateral compartment of both legs. Ten months after surgery, the patient walked with no assistance and with complete muscle function.

Conclusion Knowledge of the association between compartment syndrome and prolonged laparoscopic surgery is essential for an early diagnosis and immediate surgical treatment to avoid serious sequelae. In our patient, the good outcomes resulted from quick action, since diagnosis is often delayed. Limiting the lithotomy position to those surgical moments in which it is essential and changing the position of the legs every 2 hours during prolonged procedures can reduce the occurrence and incidence of compartment syndrome, preventing this complication.
\end{abstract}

litotomía debería limitarse a aquellos momentos de la cirugía en los que sea imprescindible, modificando la posición de las piernas cada dos horas en caso de cirugías prolongadas, para prevenir dicha complicación.

\section{Introducción}

El síndrome compartimental (SC) es una complicación infrecuente pero severa que puede aparecer tras una posición de litotomía prolongada con el uso de perneras. Su incidencia se ha descrito en 1 por cada 3.500 casos de pacientes intervenidos en dicha posición. ${ }^{1-3}$ En 1979, Leff y Shapiro ${ }^{4}$ describieron el primer caso de SC asociada a posición de litotomía.

\section{Caso Clínico}

Un paciente varón de 43 años, que, como único antecedente de interés, presentaba un Síndrome de Gilbert, fue sometido a una uretroplastia bulbar anterior tipo Palmintieri en posición habitual de litotomía durante un periodo de 6 horas. A las 2 horas de finalizar la cirugía, el paciente empezó a presentar dolor intenso y endurecimiento del compartimento anterior y lateral de ambas piernas junto con un dolor intenso para la flexión dorsal pasiva de ambos tobillos. No presentaba clínica de afectación del nervio peroneo ni dolor en el resto de los compartimentos de la pierna.

\section{Resultados}

Ante la sospecha de estar desarrollando un SC anterolateral, se midió la presión intracompartimental, que resultó en un valor de $40 \mathrm{~mm} \mathrm{Hg}$, por lo que se realizó fasciotomía bilateral urgente de ambos compartimentos, en la que se observó una disminución de la perfusión muscular, con mejoría de la misma tras apertura de la fascia (-Figura 1). Durante el proceso, el paciente presentó una elevación progresiva de la

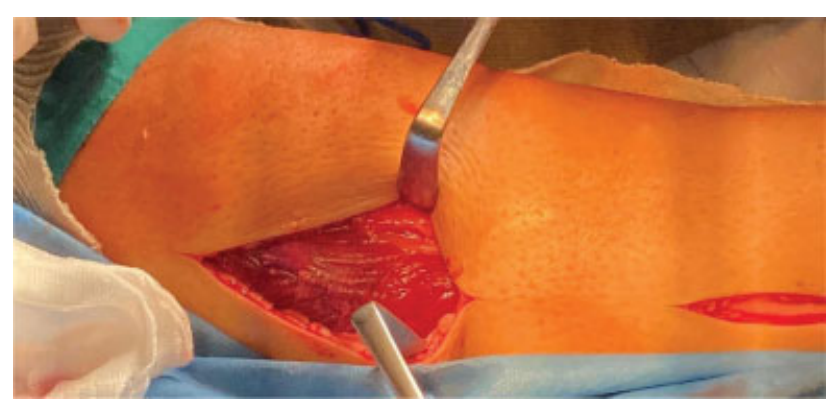

Fig. 1 Fasciotomía bilateral urgente del compartimento anterolateral de ambas piernas. Tras la apertura de la fascia, se observó una mejoría de la perfusión. 
enzima creatina quinasa (creatine kinase, $\mathrm{CK}$, en inglés) sérica (valor inicial de $109 \mathrm{U} / \mathrm{L}$, que se elevó hasta $7.689 \mathrm{U} / \mathrm{L}$ a las 12 horas de haberse realizado la fasciotomía). Se practicaron curas periódicas cada 48 horas, y se observó la viabilidad del tejido hasta su cierre definitivo a los 4 días. A las dos semanas, el paciente presentaba fatiga ligera para la deambulación sin ayuda, con tumefacción en el compartimento lateral de ambas piernas. A los diez meses de evolución, el paciente deambulaba sin ayuda y sin déficit neuromuscular.

\section{Discusión}

El caso que describimos se trata de un SC bilateral sobre piernas sanas. Normalmente está producido por causas traumáticas o vasculares; ${ }^{5}$ sin embargo, se han descrito algunos casos en la literatura científica ${ }^{2-4,6-9}$ de SCs asociados a la colocación del paciente en una posición prolongada de litotomía. El origen de dicha complicación es una elevación de la presión intracompartimental en los compartimentos de la pierna, producida por varios factores: la elevación de los miembros inferiores origina una disminución en la presión arterial (PA), que se suma a la ya causada por los agentes anestésicos; asimismo, el uso de perneras aumenta la presión en el compartimento que apoya, y la flexión de la caderas y de las rodillas provoca una disminución del retorno venoso; todos estos factortes condicionan una isquemia, que, una vez finalizada la cirugía y volviendo a su posición normal, origina una reperfusión rápida de los tejidos que se traduce en un edema y un aumento de la presión, causando un SC. ${ }^{2,5,6,8,9}$ Tal y como describe Matsen, ${ }^{10}$ la elevación de las piernas por sí misma ya provoca un aumento de la presión intracompartimenal notable, de aproximadamente $18 \mathrm{mmHg}$, por lo que dicha posición puede ser un factor muy importante para el desarrollo de esta complicación.

El diagnóstico tardío del SC provoca daños irreversibles musculares que conlleven a la disfunción de la extremidad o a la amputación del miembro, por lo que es necesario un diagnóstico precoz y una actuación quirúrgica sin demora. ${ }^{2,4,6,11,12}$ Para el diagnóstico, puede ser suficiente con la historia clínica y con la exploración física, pero, en caso de dudas, puede realizarse la medición del compartimento, en la que se confirma si la presión compartimental intracompartimental es superior o está dentro de los $30 \mathrm{mmHg}$ de diferencia respecto a la PA diastólica. ${ }^{6}$

Hasta la fecha, son pocos los $\operatorname{casos}^{8,13}$ descritos de SC bilateral tras posición de litotomía prolongada, si bien la mayoría ocurren con cirugías de duración entre 5 y 8 horas; se han descrito casos de SC tras cirugías de menos de 2 horas de duración, tal y como describen Stornelli et al. ${ }^{11}$ (duración de 90 minutos).

En cuanto a la duración de la cirugía y a la posición de litotomía, Yamamoto et al. ${ }^{2}$ refiere la complicación de su caso pasadas 16 horas, presentándose con una clínica más intensa y una mayor elevación de la CK sérica, similar al caso presentado por GarcíaGermán et $\mathrm{al}^{7}{ }^{7}$ siendo esta mucho menos florida. Del mismo modo, Oman et al. ${ }^{14}$ presentan un caso de SC que surge a la hora de finalizarse una cirugía ginecológica de seis horas de duración, similar al caso que presentamos nosotros, realizando fasciotomía del compartimento posterior, pero precisando una cirugía de revisión al día siguiente, y completando la fasciotomía de los tres compartimentos restantes por persistencia y empeoramiento de la clínica, quedando como secuela parestesias en el tobillo y pie izquierdos. Estos casos, similares al nuestro en cuanto a la duración de la cirugía, tienen una mayor severidad de la complicación, quizás por la duración de los síntomas. En nuestro caso, el paciente ha estado estable hemodinámicamente, sin presentar alteraciones analíticas notorias ni de los parámetros renales, así como una elevación de la CK no superior a los $8.000 \mathrm{U} / \mathrm{L}$, a diferencia del resto de casos presentados, ${ }^{2,3,7-9,11}$ que llegan a alcanzar cifras de hasta 142.000 U/L. Del mismo modo, durante el seguimiento, el paciente presentó una mejoría rápidamente progresiva, sin déficits neurovasculares residuales, tal y como el caso presentado por Stornelli et al. ${ }^{11}$ Esto quizá fue debido a un diagnóstico y actuación precoz por nuestra parte, con una rápida recuperación y mejoría en el pronóstico de la extremidad.

Hasta la fecha, únicamente se han descrito casos de SC crónicos tratados mediante fasciotomía mínimamente invasiva, ${ }^{15,16}$ tal y como presentamos el nuestro, por lo que podría ser una alternativa ante un SC agudo de poco tiempo de evolución o en estadio precoz.

\section{Conclusión}

El conocimiento de la asociación del SC y la cirugía laparoscópica prolongada es esencial para un diagnóstico precoz y un tratamiento quirúrgico inmediato, para evitar graves secuelas. Los buenos resultados de nuestro paciente se deben a la rápida actuación, ya que normalmente se suele demorar. Para evitar su aparición o disminuir su incidencia, la posición de litotomía debe limitarse a aquellos momentos de la cirugía en los que sea imprescindible, modificando la posición de las piernas cada 2 horas en caso de cirugías prolongadas, para prevenir dicha complicación. No obstante, se ha propuesto el empleo de nuevas botas de pernera que parecen disminuir la incidencia de dicha complicación. En estas perneras, las piernas del paciente se apoyan sobre una superficie revestida por un material blando sujeta por bandas ajustables; además, permiten la posibilidad de modificar la posición de los miembros inferiores durante el acto quirúrgico, y también el apoyo de la planta del pie, por lo que se reducen las posibilidades de compresión del nervio peroneo y del desarrollo de SC.

\section{Conflicto de Intereses}

Los autores declaran que no hay conflicto de intereses.

\section{Referencías}

1 Mumtaz FH, Chew H, Gelister JS. Lower limb compartment syndrome associated with the lithotomy position: concepts and perspectives for the urologist. BJU Int 2002;90(08): 792-799

2 YamamotoT, Fujie A, Tanikawa H, Funayama A, Fukuda K. Bilateral Well Leg Compartment Syndrome Localized in the Anterior and Lateral Compartments following Urologic Surgery in Lithotomy Position. Case Rep Orthop 2018;2018:2328014

3 Nishino M, Okano M, Kawada J, Kim Y, Yamada M, Tsujinaka T. Well-leg compartment syndrome after laparoscopic low anterior resection for lower rectal cancer in the lithotomy position: A case 
report. Asian J Endosc Surg ISSN 1758-5902. 2017https:// pubmed.ncbi.nlm.nih.gov/28677871/

4 Leff RG, Shapiro SR. Lower extremity complications of the lithotomy position: prevention and management. J Urol 1979; 122(01):138-139

5 von Keudell AG, Weaver MJ, Appleton PT, et al. Diagnosis and treatment of acute extremity compartment syndrome. Lancet 2015;386(10000):1299-1310

6 Christoffersen JK, Hove LD, Mikkelsen KL, Krogsgaarsd MR. Well Leg Compartment Syndrome After Abdominal Surgery. World J Surg 2017;41(02):433-438

7 García-Germán Vazquez D, Aragonés Maza P, Zorzo Codes C, López Gonzalez D. Sindrome compartimental en pierna sana, una complicación de la cirugía laparoscópica prolongada. Trauma Fund MAPFRE 2013;24(04):217-220

8 Dos Reis JMC, Queiroz LJM, Mello PF, Teixeira RKC, Gonçalves FA. Bilateral compartment syndrome of the lower limbs after urological surgery in the lithotomy position: a clinical case. J Vasc Bras 2019;18:e20180117

9 Enomoto T, Ohara Y, Yamamoto M, Oda T, Ohkohchi N. Well leg compartment syndrome after surgery for ulcerative colitis in the lithotomy position: A case report. Int J Surg Case Rep 2016; 23:25-28
10 Matsen FA III. A practical approach to compartmental syndromes. Part I. Definition, theory, and pathogenesis. Instr Course Lect 1983;32:88-92

11 Stornelli N, Wydra FB, Mitchell JJ, Stahel PF, Fabbri S. The dangers of lithotomy positioning in the operating room: case report of bilateral lower extremity compartment syndrome after a 90minutes surgical procedure. Patient Saf Surg 2016;10:18

12 Mortensen SJ, Zhang D, Mohamadi A, et al. Predicting factors of muscle necrosis in acute compartment syndrome of the lower extremity. Injury 2020;51(02):522-526

13 Simms MS, Terry TR. Well leg compartment syndrome after pelvic and perineal surgery in the lithotomy position. Postgrad Med J 2005;81(958):534-536

14 Oman SA, Schwarz D, Muntz HG. Lower limb compartment syndrome as a complication of radical hysterectomy. Gynecol Oncol Rep 2016;16:39-41

15 Mouhsine E, Garofalo R, Moretti B, Gremion G, Akiki A. Two minimal incision fasciotomy for chronic exertional compartment syndrome of the lower leg. Knee Surg Sports Traumatol Arthrosc 2006;14(02): 193-197

16 Maffulli N, Loppini M, Spiezia F, D’Addona A, Maffulli GD. Single minimal incision fasciotomy for chronic exertional compartment syndrome of the lower leg. J Orthop Surg Res 2016;11(01):61 